University of Nebraska - Lincoln

DigitalCommons@University of Nebraska - Lincoln

$5-14-2008$

\title{
Magnetic behavior of melt-spun gadolinium
}

\author{
P. M. Shand \\ University of Northern lowa \\ J. G. Bohnet \\ University of Northern lowa \\ Jeffrey E. Shield \\ University of Nebraska - Lincoln, jshield@unl.edu \\ D. Schmitter \\ University of Nebraska - Lincoln \\ G. Shelburne \\ University of Nebraska - Lincoln
}

See next page for additional authors

Follow this and additional works at: https://digitalcommons.unl.edu/physicslesliepelecky

Part of the Physics Commons

Shand, P. M.; Bohnet, J. G.; Shield, Jeffrey E.; Schmitter, D.; Shelburne, G.; and Leslie-Pelecky, Diandra, "Magnetic behavior of melt-spun gadolinium" (2008). Diandra Leslie-Pelecky Publications. 21. https://digitalcommons.unl.edu/physicslesliepelecky/21

This Article is brought to you for free and open access by the Research Papers in Physics and Astronomy at DigitalCommons@University of Nebraska - Lincoln. It has been accepted for inclusion in Diandra Leslie-Pelecky Publications by an authorized administrator of DigitalCommons@University of Nebraska - Lincoln. 


\section{Authors}

P. M. Shand, J. G. Bohnet, Jeffrey E. Shield, D. Schmitter, G. Shelburne, and Diandra Leslie-Pelecky 


\title{
Magnetic behavior of melt-spun gadolinium
}

\author{
P. M. Shand and J. G. Bohnet \\ Department of Physics, University of Northern Iowa, Cedar Falls, Iowa 50614-0150, USA \\ J. Goertzen and J. E. Shield \\ Department of Mechanical Engineering and Nebraska Center for Materials and Nanoscience, University of Nebraska, \\ Lincoln, Nebraska 68588, USA
}

\author{
D. Schmitter, G. Shelburne, and D. L. Leslie-Pelecky \\ Department of Physics and Astronomy and Nebraska Center for Materials and Nanoscience, University of Nebraska, Lincoln, Nebraska \\ 68588, USA \\ (Received 21 September 2007; revised manuscript received 21 March 2008; published 14 May 2008)
}

\begin{abstract}
Melt-spun Gd is a structurally inhomogeneous system consisting of crystalline grains with an average size of $24 \pm 3 \mathrm{~nm}$ that are separated by an amorphous interphase. This system exhibits a depression of $T_{C}$ $(289.70 \pm 0.01 \mathrm{~K})$ relative to bulk $\mathrm{Gd}(293 \mathrm{~K})$. The effective critical exponents $\left(\beta_{\text {eff }}=0.389 \pm 0.017, \gamma_{\text {eff }}\right.$ $=1.300 \pm 0.014$, and $\delta=4.32 \pm 0.02$ ) and critical amplitudes indicate that for the reduced-temperature range in this work, the paramagnetic-to-ferromagnetic transition is consistent with the isotropic dipolar universality class shown by bulk Gd. There is, however, evidence of enhanced anisotropy in the critical behavior of ms-Gd. Increasing random anisotropy in the intergrain regions with decreasing temperature below $T_{C}$ diminishes the coupling between the ferromagnetically ordered grains and produces a previously unobserved low-temperature peak in the imaginary part of the ac susceptibility. The random-anisotropy model provides a good description of the approach to saturation, which may result from the ferromagnetic correlation length becoming comparable to the anisotropy correlation length in the strong-field regime of the model.
\end{abstract}

DOI: 10.1103/PhysRevB.77.184415

PACS number(s): 75.50.-y, 75.30.Kz, 75.30.Gw

\section{INTRODUCTION}

Disordered magnetic materials exhibit a rich variety of magnetic and electronic properties, ${ }^{1-8}$ such as soft magnetism and giant magnetoresistance, that find applications in information processing and communications. ${ }^{9}$ The multiplicity of phases and behaviors (e.g., colossal magnetoresistance) found in strongly correlated electron systems such as manganites is in part due to "clustered states"-nanoscale regions arising from competing phases and disorder-induced inhomogeneity. ${ }^{8}$ Similarly, competition between ordered (ferromagnetic or ferrimagnetic) nanoscale grains and disordered surrounding grain-boundary regions produces complex and varied behaviors in systems with significant volume fractions of grain-boundary regions. ${ }^{4}$ Exchange coupling of grains can produce a very soft magnetic behavior, while decoupled grains lead to a single-domain hard magnetic behavior.

$\mathrm{Gd}$ is an excellent candidate for studying the effects of the nanostructure on magnetic behavior because the ferromagnetism arises from well-localized spin-only $\mathrm{Gd}$ ions (although there is some polarization of the conduction band), and the paramagnetic-to-ferromagnetic (PM-FM) transition temperature $T_{C}$ for bulk crystalline $\mathrm{Gd}(\approx 293 \mathrm{~K})$ is close to room temperature. The critical behavior near a continuous phase transition in a pure system depends on the spatial and spin dimensionalities, the range of the spin-spin interaction, and the strengths of coherent and random anisotropies. Structural inhomogeneity may affect one or more of these factors and thus change the transition's universality class. In addition, the observation of low-temperature spin freezing in nanocrystalline $\mathrm{Fe},{ }^{6,7} \mathrm{FeRh},{ }^{5}$ and other alloys suggests that similar effects may be present in structurally inhomogeneous Gd. Thus, we have performed structural and magnetic measurements on a sample of melt-spun Gd (ms-Gd). To obtain comprehensive understanding of the magnetic behavior, the temperature range of the magnetic measurements encompassed the paramagnetic region $\left(T>T_{C}\right)$, the paramagneticto-ferromagnetic phase transition $\left(T \approx T_{C}\right)$, and the lowtemperature region below the transition temperature $(T$ $\left.<T_{C}\right)$.

\section{EXPERIMENTAL DETAILS}

Melt-spun Gd ribbons were prepared by first arc melting gadolinium $(99.9 \%)$ pieces multiple times in a copper crucible and an argon atmosphere. The resulting ingot was placed in a cylindrical quartz crucible with an orifice at the bottom and was melt spun in an Ar atmosphere onto a cooled copper disk rotating with a tangential speed of $40 \mathrm{~m} / \mathrm{s}$. Pieces of the melt-spun ribbon were mounted with silicone vacuum grease on a zero-background holder for x-ray diffraction (XRD). Transmission electron microscope (TEM) samples of the melt-spun ribbons were prepared by gluing the ribbon pieces to $\mathrm{Cu}$ grids and then ion milling.

dc magnetization and ac susceptibility measurements were performed with a Quantum Design PPMS system with the ACMS option. The sample consisted of several ribbon pieces, each with a thickness of $\sim 0.04 \mathrm{~mm}$, a width of $\sim 2 \mathrm{~mm}$, and a length of $\sim 5 \mathrm{~mm}$. The total mass of the sample was $11.92 \mathrm{mg}$. The ribbons were sealed in polyethylene bags in an Ar atmosphere to minimize oxidation. The longest dimension of the ribbons was oriented along the field 
direction to minimize the demagnetization factor. Isothermal magnetization-versus-internal magnetic field data were collected at applied (external) fields between 100 Oe and 60 kOe. Magnetization isotherms were taken at temperatures $286 \mathrm{~K} \leq T \leq 294 \mathrm{~K}$, with the temperature held stable to within $\pm 0.02 \mathrm{~K}$. The ac susceptibility $\chi_{\text {ac }}$ was simultaneously measured with the dc magnetization $(M)$, which enabled an accurate calculation of the internal magnetic field and internal susceptibility at each temperature $(286 \mathrm{~K} \leq T$ $\leq 305 \mathrm{~K}$ ), with increments of $0.5 \mathrm{~K} . \chi_{\mathrm{ac}}(T)$ was measured for superimposed dc bias fields between 600 and 3000 Oe. The ac driving field used in these measurements was 3 Oe at a frequency of $1000 \mathrm{~Hz}$. Magnetic isotherms and $\chi_{\mathrm{ac}}(T)$ data were collected by first warming the sample to $320 \mathrm{~K}$ (well above $T_{C}$ for bulk crystalline $\mathrm{Gd}$ ) to demagnetize it, cooling it in zero applied field down to $286 \mathrm{~K}$, and then taking data while warming. (The "zero" field for the system was between 1 and $10 \mathrm{Oe}$, due to the remanent field of the magnet and the earth's field.) Care was taken in all cases to ensure that the system reached thermal equilibrium after each change in temperature.

The internal magnetic field and internal susceptibility were computed by first estimating the effective demagnetization factor $N$ for the sample. This was done by warming the sample to a temperature significantly above $T_{C}$ and then cooling it in zero field to a temperature just below $T_{C}$. The dc magnetization was then measured in applied fields $H_{\text {app }}$ $<20$ Oe (corrected for the magnet's remanent field) and $N$ was approximated as the reciprocal of the initial dc susceptibility, yielding a value of 0.59 (Gaussian units) by using the measured density of $7.70 \mathrm{~g} / \mathrm{cm}^{3}$. The internal quantities were calculated from $H_{\text {int }}=H_{\text {app }}-N M$ and $\chi_{\text {int }}^{\prime}=\chi_{\text {ext }}^{\prime} /(1$ $-N \chi_{\mathrm{ext}}^{\prime}$ ). Because the samples were not oriented such that the field was exactly along the $c$ axis, the estimated demagnetization factor is affected by the variation in the anisotropy of Gd with temperature and thus is larger than the purely geometric demagnetization factor. ${ }^{10}$

ac susceptibility and dc magnetization were also measured at temperatures down to $5 \mathrm{~K}$ to investigate the lowtemperature behavior. For the ac susceptibility measurements, ac fields of 0.1 and 1.0 Oe were used and the frequency was varied between $159 \mathrm{~Hz}$ and $10 \mathrm{kHz}$.

\section{EXPERIMENTAL RESULTS}

\section{A. Structural measurements}

The x-ray diffraction pattern for a melt-spun Gd ribbon is shown in Fig. 1. The peak positions are consistent with hcp $\mathrm{Gd}$, as indicated by the solid lines. Within the resolution of the instrument, no other Gd phases or impurities were detected. Note that the peaks are slightly displaced relative to bulk hcp Gd, which is indicative of the presence of uniform strain in the lattice structure. Characteristics of the microstructure were obtained by analyzing the peak widths as a function of diffraction angle and utilizing a Williamson-Hall plot. By utilizing the Scherrer formula, the grain size was determined to be $24 \pm 3 \mathrm{~nm}$. However, a TEM image (Fig. 2) revealed a grain size of approximately $160 \mathrm{~nm}$. The large discrepancy between the XRD and TEM values is probably

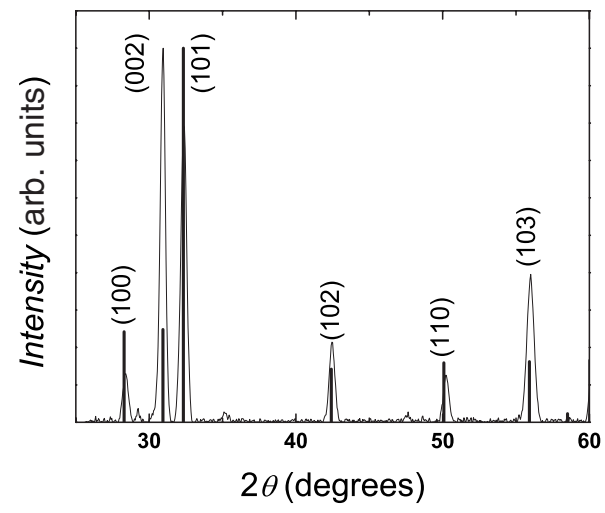

FIG. 1. XRD scan of a melt-spun Gd ribbon. The solid lines are the expected positions for bulk hcp-Gd.

due to the limited sampling region allowed by TEM, which precludes a statistically valid grain size measurement, especially in this case wherein so few grains were imaged. Analysis by XRD provides an overall average of the grain size. The TEM image in Fig. 2 shows the intergrain regions with an average width of $\sim 10 \mathrm{~nm}$. From extended x-ray absorption fine structure (EXAFS) spectroscopy measurements, ${ }^{11}$ we estimate these regions to be $[15( \pm 5)] \%$ of the sample volume. The EXAFS measurements also indicate that the intergrain regions are amorphous. More details on the EXAFS measurements will be presented in a forthcoming paper.

\section{B. Magnetic measurements}

\section{Paramagnetic regime}

Figure 3 shows the reciprocal of the ac susceptibility versus temperature for temperatures between 300 and $350 \mathrm{~K}$. The Curie-Weiss law is followed reasonably well for $T$ $>320 \mathrm{~K}$. A linear fit to the $T>320 \mathrm{~K}$ data yields the CurieWeiss temperature $\Theta=296.5 \mathrm{~K}$ and the effective number of Bohr magnetons $p_{\text {eff }}=8.14$. Deviation from linearity is observed below $320 \mathrm{~K}$, which is significantly higher than the PM-FM transition temperature $T_{C}$.

\section{Critical region}

The behavior of a ferromagnet in the vicinity of the phase transition is often described by the Arrott-Noakes magnetic equation of state, ${ }^{12}$

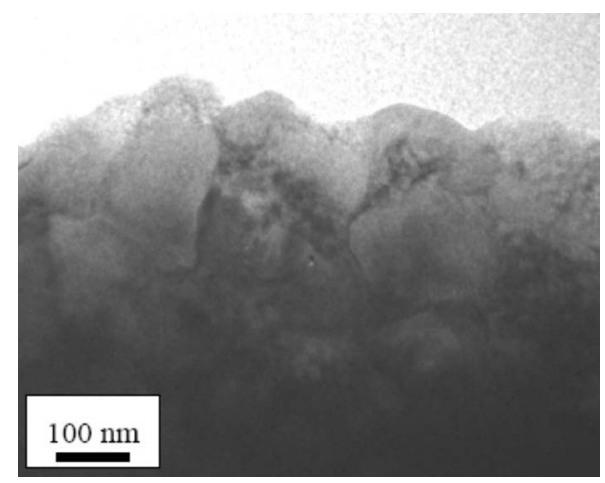

FIG. 2. TEM image of a melt-spun Gd sample. The average grain size is $160 \mathrm{~nm}$. 


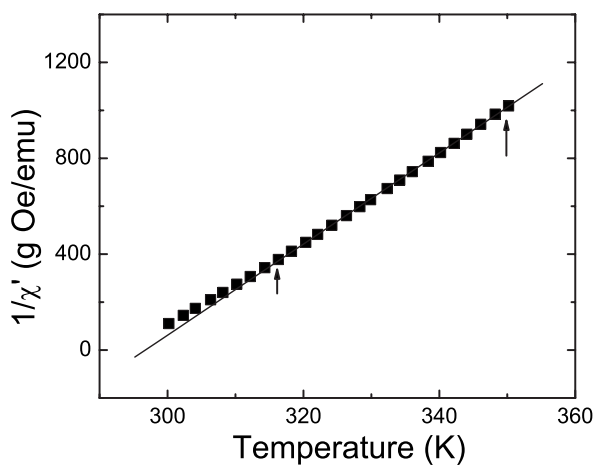

FIG. 3. Inverse ac susceptibility versus temperature $T$ for $300 \mathrm{~K} \leq T \leq 350 \mathrm{~K}$. The arrows indicate the range of the linear fit.

$$
M^{1 / \beta}=a \varepsilon+b(H / M)^{1 / \gamma},
$$

where $H$ is the internal magnetic field, $a$ and $b$ are coefficients independent of (or at least weakly dependent on) the reduced critical temperature $\varepsilon=\left(T-T_{C}\right) / T_{C}$, and $\beta$ and $\gamma$ are critical exponents. Equation (1) is a generalization of the magnetic equation of state obtained from the Landau meanfield theory for which $\beta=0.5$ and $\gamma=1$, so a mean-field system should produce a set of parallel lines when $M^{2}$ versus $H / M$ is plotted for isotherms spanning $T_{C}$. Our data show a strong curvature in all isotherms up to the highest applied field $(60 \mathrm{kOe})$, indicating that a mean-field description of the critical behavior of the ms-Gd system is not appropriate.

A system with a non-mean-field critical behavior requires an Arrott-Noakes (AN) plot, in which isotherms spanning $T_{C}$ will generate a set of parallel straight lines when the data are plotted as $M^{1 / \beta}$ versus $(H / M)^{1 / \gamma}$. The critical isotherm will pass through the origin for the appropriate values of $\beta$ and $\gamma$. In addition to satisfying Eq. (1), $\beta$ and $\gamma$ also obey powerlaw relations derived from the renormalization group theory in the critical regime. ${ }^{13}$ The exponent $\beta$ is the critical exponent for the spontaneous (zero-field) magnetization $M_{S}$ and is defined (to leading order) by

$$
M_{S}(T)=M_{0} B(-\varepsilon)^{\beta}, \quad \varepsilon<0,
$$

where $M_{0}=N g \mu_{B} S$ is the zero-temperature saturation magnetization and $B$ is a critical amplitude. Equation (2) is an approximation because correction-to-scaling terms have been omitted, so the $\beta$ and $B$ values determined by using Eq. (2) are in reality effective quantities, which will generally depend on the temperature unless one is extremely close to the critical point, i.e., within the asymptotic critical region.

The exponent $\gamma$ is the critical exponent for the zero-field or initial susceptibility. It is defined by

$$
\chi_{i}(T)=\chi_{0} \Gamma \varepsilon^{-\gamma}, \quad \varepsilon>0,
$$

where $\Gamma$ is a critical amplitude, $\chi_{0}=M_{0} / H_{0}$, and $H_{0}$ $=k_{B} T_{C} / g \mu_{B} S$. As with Eq. (2), the correction-to-scaling terms have been neglected in Eq. (3), assuming that one is operating within the asymptotic critical region.

To obtain values of $\beta$ and $\gamma$ for ms-Gd, data for $286 \mathrm{~K}$ $\leq T \leq 294 \mathrm{~K}$ were plotted according to Eq. (1) by using $\beta$ $=0.365$ and $\gamma=1.386$, which are values for the short-range three-dimensional (3D) Heisenberg model. ${ }^{14}$ There were de-

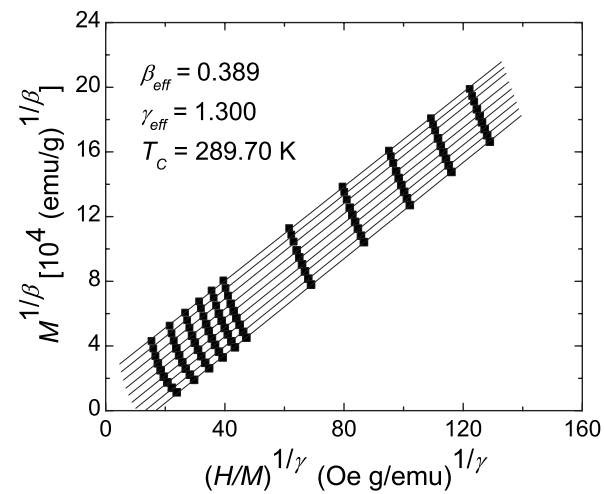

FIG. 4. Arrott-Noakes plot for $\beta_{\text {eff }}=0.389, \gamma_{\text {eff }}=1.300$, and $T_{C}$ $=289.70 \mathrm{~K}$. The highest external field used was $60 \mathrm{kOe}$. The temperature range for the data was $286 \mathrm{~K} \leq T \leq 294 \mathrm{~K}$.

viations from a near-straight-line behavior at low fields, which are commonly seen in AN plots. These low-field points were excluded from the subsequent data analysis. The culled magnetization data were then fitted according to Eq. (1) by using $\beta$ and $\gamma$ (as well as $a \varepsilon$ and $b$ ) as fitting parameters. The values of $\beta$ and $\gamma$ that were thus obtained were used to generate an AN plot, from which the spontaneous magnetization $M_{S}$ at each temperature was obtained. The critical temperature was obtained from a straight-line fit to $\left(M_{S}\right)^{1 / \beta}$ versus $T$ for the lowest temperatures in the range [see Eq. (2)]. The inverse zero-field susceptibility $\chi_{i}^{-1}$ for each temperature was obtained from the intercepts on the $(H / M)^{1 / \gamma}$ axis of the AN plot $\left(T>T_{C}\right)$. Refined estimates of $\beta$ and $\gamma$ were then obtained from the slopes of log-log plots of $M_{S}$ versus $\varepsilon$ and $\chi_{i}^{-1}$ versus $\varepsilon$ [see Eqs. (2) and (3)]. The refined values of $\beta$ and $\gamma$ were used to generate new AN plots. The entire procedure was iterated until the new and old values of the exponents were the same within error limits. The final AN plot, which is shown in Fig. 4, yields $T_{C}$ $=289.70 \pm 0.01 \mathrm{~K}, \quad \beta_{\text {eff }}=0.389 \pm 0.017, \quad$ and $\gamma_{\text {eff }}$ $=1.300 \pm 0.014$. These exponents are labeled effective exponents because of the omission of correction-to-scaling terms. For the determination of $\beta_{\text {eff }}$, the reduced-temperature range was $2.4 \times 10^{-3} \leq|\varepsilon| \leq 1.3 \times 10^{-2}$. For $\gamma_{\mathrm{eff}}, 1.0 \times 10^{-3} \leq \varepsilon$ $\leq 1.5 \times 10^{-2}$.

A third exponent characterizing the critical behavior is the critical-isotherm exponent $\delta$, which is defined by

$$
H=H_{0} D\left(M / M_{0}\right)^{\delta}, \quad T=T_{C},
$$

where $D$ is a critical amplitude. There is no magnetizationversus-internal field data for an isotherm exactly at $T_{C}$; however, we may estimate $\delta$ by using

$$
T_{C}=T-A\left[\delta\left(T_{C}\right)-\delta(T)\right],
$$

where $A$ is sample-dependent constant. ${ }^{14}$ Empirically, ferromagnets have been found to obey Eq. (5) in the temperature range $T_{C} \pm 1 \mathrm{~K}$. Log-log plots of $M$ versus $H$ at 289 and 290 $\mathrm{K}$ (Fig. 5) produced two values for $\delta(T)$. We then used Eq. (5) to obtain $\delta\left(T_{C}\right)=\delta=4.32 \pm 0.02$. This value agrees well with that expected from the Widom scaling relation ${ }^{15} \delta=1$ $+(\gamma / \beta)$, which yields $\delta=4.34 \pm 0.19$ when using the experimental values of $\beta_{\text {eff }}$ and $\gamma_{\text {eff }}$ given above. (We have not used 


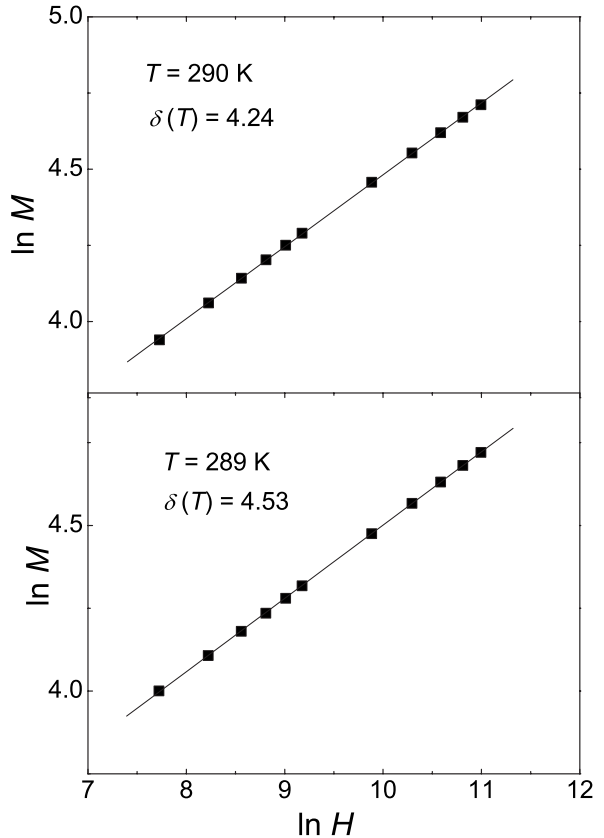

FIG. 5. Plots of $\ln M$ versus $\ln H$ for $T=289$ and $290 \mathrm{~K}$. Equation (5) in the text was used to find the value of $\delta$ at $T_{C}$ $=289.70 \mathrm{~K}$.

the "effective" notation for $\delta$ because it is determined at $T_{C}$ and so is presumably asymptotic.)

We have also obtained an estimate of $\gamma$ by measuring the ac susceptibility in the critical region with a superimposed dc bias field. Figure 6 shows the magnitude of the ac susceptibility $\chi_{\mathrm{ac}}(T)$ measured in different static external bias fields $H_{\mathrm{dc}}$. In the vicinity of the critical point, increasing values of $H_{\mathrm{dc}}$ correspondingly suppress the main (Hopkinson) peak in $\chi_{\mathrm{ac}}(T)$, eventually revealing a secondary maximum produced by critical-point fluctuations. ${ }^{16-18}$ The locus of the secondary maxima represents a crossover line from a thermally dominated to a field-dominated behavior. One can extract $\gamma$ from these data by using

$$
\chi(T, H)=\left(\frac{\partial M}{\partial H}\right)=\varepsilon^{-\gamma} G\left(H / \varepsilon^{\beta+\gamma}\right),
$$

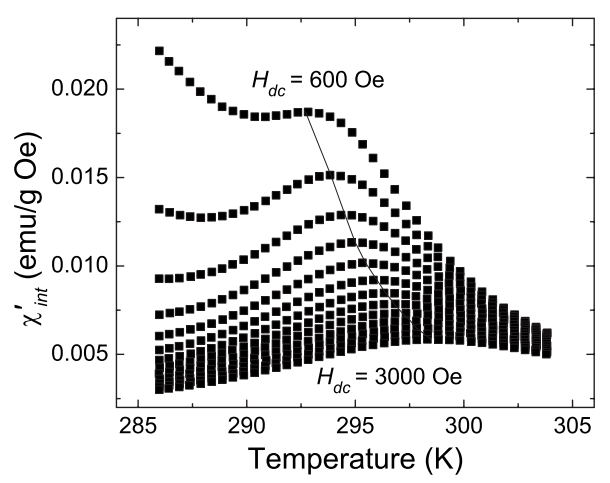

FIG. 6. Magnitude of the ac susceptibility $\chi_{\mathrm{ac}}(T)$ measured in different static external bias fields $\left(600\right.$ Oe $\leq H_{\mathrm{dc}} \leq 3000$ Oe $)$ in the vicinity of the Curie temperature. The measuring frequency was $1000 \mathrm{~Hz}$ and the ac field strength was 3 Oe. The line passes through the maximum for each $H_{\mathrm{dc}}$ value.

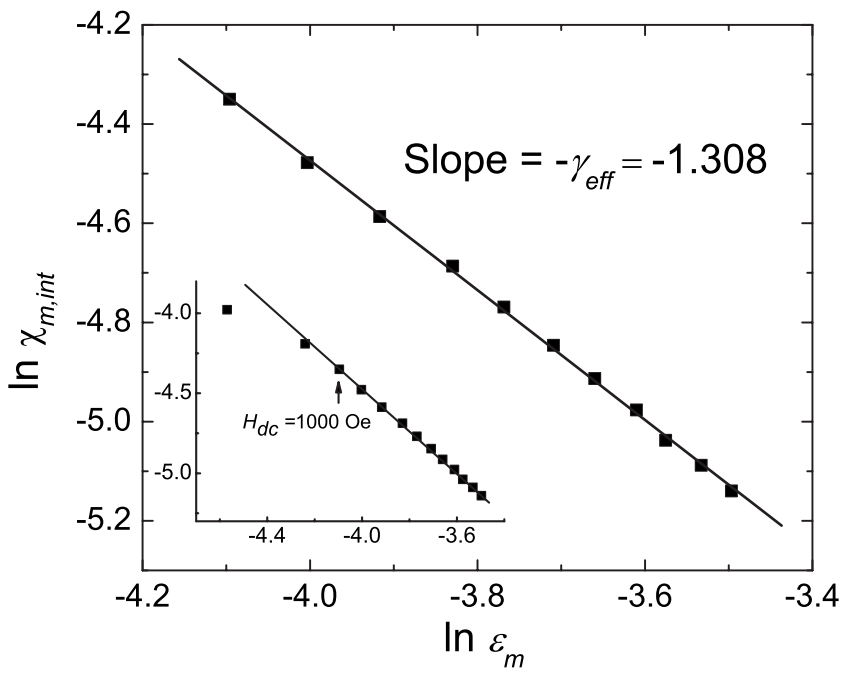

FIG. 7. Natural logarithm of zero-field susceptibility evaluated at the critical maximum $\chi\left(T_{m}, H\right)$ versus natural logarithm of the reduced temperature evaluated at the maximum. The inset shows the additional data for the lowest two bias fields (600 and $800 \mathrm{Oe})$ and the deviation from linearity.

where $H$ is the internal field and $G$ is a scaling function. Since $G$ is a universal function of its argument, if the data are scaled according to the argument of $G$, all of the data will collapse onto a single curve. If we write the reduced temperature at the maximum in $\chi(T, H)$ as $\varepsilon_{m}=\left(T_{m}-T_{C}\right) / T_{C}$, then we have $H / \varepsilon_{m}^{\beta+\gamma}=$ const, which leads to $\varepsilon_{m} \propto H^{1 /(\beta+\gamma)}$. Since the argument in Eq. (6) is constant at the susceptibility maximum, one finds that

$$
\chi\left(T_{m}, H\right) \propto \varepsilon_{m}^{-\gamma} .
$$

The exponent $\gamma$ (actually $\gamma_{\text {eff }}$ ) can be determined from a $\log -\log$ plot of the zero-field susceptibility evaluated at the critical maximum versus the reduced temperature evaluated at the maximum, such as that shown in Fig. 7, by using $\chi_{\mathrm{ac}}$ as the experimental realization of $\chi$. A linear fit using external bias fields $H_{\mathrm{dc}} \geq 1000 \mathrm{Oe}$ and $T_{C}=289.70 \mathrm{~K}$ (obtained from the Arrott-Noakes analysis) gives $\gamma_{\mathrm{eff}}=1.308 \pm 0.012$ for $1.7 \times 10^{-2} \leq \varepsilon \leq 3.0 \times 10^{-2}$. This is in good agreement with the value $\gamma_{\text {eff }}=1.300 \pm 0.014$ obtained from the dc magnetization data. $T_{C}$, estimated from the $y$ intercept of a linear fit to a $T_{m}$ versus $H^{1 /(\beta+\gamma)}$ graph, yields $T_{C}=290.2 \pm 0.7 \mathrm{~K}$, when using $\gamma=1.308$ and $\beta=0.389$. The inset of Fig. 7 shows significant deviation from the straight-line fit for the smallest bias fields $\left(H_{\mathrm{dc}}=600\right.$ and $\left.800 \mathrm{Oe}\right)$. As the demagnetization field is no more than $\sim 10 \%$ of the external field, the limited accuracy in the determination of the demagnetization constant is not responsible for the quite sharp deviation from linearity at low bias fields.

\section{Ferromagnetic regime and low-temperature behavior}

The zero-field-cooled (ZFC) and field-cooled (FC) magnetization data as a function of temperature are shown in Fig. 8. The applied magnetic field was 100 Oe. The peak in the ZFC magnetization occurs at $120 \mathrm{~K}$ and the FC magnetization separates from the ZFC magnetization at the ZFC peak. 


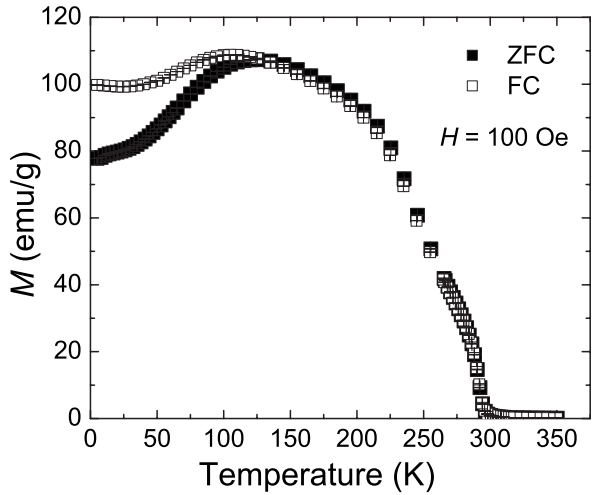

FIG. 8. ZFC and FC magnetizations as a function of temperature. The applied magnetic field was $100 \mathrm{Oe}$. At temperatures above the peak, the ZFC magnetization is slightly higher than the FC magnetization, but their difference is comparable to the uncertainty.

The occurrence of the ZFC/FC bifurcation point at the peak is typically observed in spin glasses (SGs) and re-entrant spin glasses (RSGs) but not in superparamagnets (in which the junction usually occurs at a much higher temperature than the peak). ${ }^{19,20}$ However, a ZFC/FC bifurcation similar to that exhibited by ms-Gd was also observed in several perovskite ferromagnets. ${ }^{21,22}$ In these materials, the ZFC/FC irreversibility is not due to magnetic glassiness, but to the effects of anisotropy in the ferromagnetic state. Note that the ZFC/FC irreversibility exhibited by a ferromagnetic material is also attributed to domain freezing. ${ }^{23}$

The real part of the external (i.e., not corrected for demagnetization) ac susceptibility as a function of the temperature $\left[\chi_{\text {ext }}^{\prime}(T)\right]$ of ms-Gd for $5 \mathrm{~K} \leq T \leq 350 \mathrm{~K}$ at $3000 \mathrm{~Hz}$ and ac field amplitudes of 0.1 and 1.0 Oe is shown in Fig. 9. The following aspects of these data are noteworthy: (i) There is a change in the slope ("shoulder") close to, but somewhat lower than, $T_{C}$; (ii) The peak located at $\sim 200 \quad \mathrm{~K}$ is somewhat flattened, which could indicate that $\chi_{\mathrm{ext}}^{\prime}(T)$ is demagnetization limited over the temperature range of the peak; (iii) $\chi_{\text {ext }}^{\prime}(T)$ at the peak is more than twice its value at $5 \mathrm{~K}$, that is, $\chi_{\text {ext }}^{\prime}(T)$ quite sharply falls off with decreasing temperature below the peak; and (iv) $\chi_{\text {ext }}^{\prime}(T)$ is practically independent of ac driving field for $H_{\mathrm{ac}}=0.1$ Oe and $1.0 \mathrm{Oe}$.

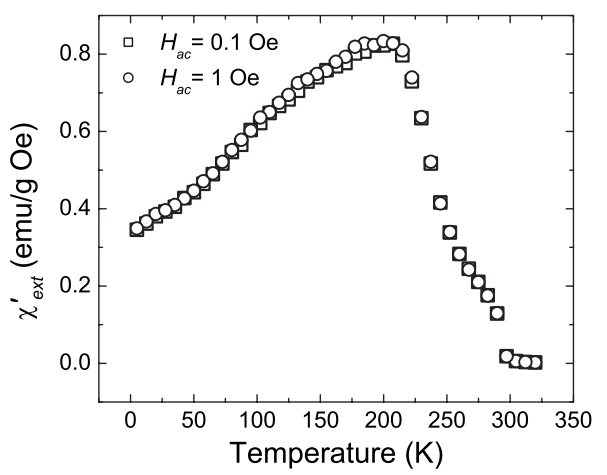

FIG. 9. Real parts of the external ac susceptibility as a function of temperature $\left[\chi_{\text {ext }}^{\prime}(T)\right]$ of ms-Gd for $5 \mathrm{~K} \leq T \leq 350 \mathrm{~K}$ for two different values of ac field amplitude $\left(H_{\mathrm{ac}}=0.1\right.$ and 1.0 Oe). The frequency is $3000 \mathrm{~Hz}$.
The shoulder close to $T_{C}$ observed in our $\chi_{\text {ext }}^{\prime}(T)$ data was not observed in previous measurements of polycrystalline $\mathrm{Gd}$ (Refs. 24 and 25); however, a shoulder was visible in measurements by Kaul and Srinath ${ }^{10}$ on a cylindrical singlecrystal Gd sample for which the cylinder axis (along which the magnetic field was applied) was out of alignment with the crystalline $c$ axis by a few degrees. ${ }^{26}$ If the field is perfectly aligned with the $c$ axis, $\chi_{\text {ext }}^{\prime}(T)$ steeply rises to the demagnetization limit at the transition, with no deviation. ${ }^{10}$ The enhanced strength of the (002) reflection in the XRD pattern of Fig. 1 indicates texturing with the $c$ axis of the grains preferentially aligned normal to the plane of the ribbons. ${ }^{11}$ Thus, the applied field in our magnetic measurements preferentially lies in the basal plane of the hexagonal unit cell, i.e., along the hard direction. The shoulder we observe in $\chi_{\mathrm{ext}}^{\prime}(T)$ is therefore due to the spatial averaging of the temperature-dependent anisotropy, with the applied field along the length of our melt-spun ribbons.

The peak and subsequent significant decrease in $\chi_{\text {ext }}^{\prime}(T)$ with decreasing temperature were previously observed in the ac susceptibility ${ }^{24}$ and permeability ${ }^{25}$ of polycrystalline $\mathrm{Gd}$. The variation in the permeability was explained in terms of the orientation of the easy axis as a function of temperature. The maximum in the permeability (and, hence, also $\chi_{\mathrm{ac}}$ ) occurs at nearly the same temperature $(\sim 180 \mathrm{~K})$ as the greatest departure of the easy axis from alignment with the local $c$ axis. The minimum in the coercivity of Gd and the passage through zero of the uniaxial anisotropy constant $K_{1}$ also occur quite close to the temperature of the permeability maximum. The coercivity quite rapidly rises at temperatures below the minimum, which is consonant with the decrease in permeability and susceptibility. In Gd single crystals, $\chi_{\text {ext }}^{\prime}(T)$ is demagnetization limited immediately below the spinreorientation temperature $\left(T_{\mathrm{SR}} \sim 230 \mathrm{~K}\right)$ when the applied field is either parallel or perpendicular to the $c$ axis. ${ }^{10}$ Furthermore, $\chi_{\mathrm{ext}}^{\prime}(T)$ decreases from the demagnetization-limited value as $T$ is lowered well below $T_{\mathrm{SR}}$.

As mentioned above, $\chi_{\text {ext }}^{\prime}(T)$ for ms-Gd is independent of $H_{\mathrm{ac}}$ for driving fields $<1$ Oe. This indicates that domainwall displacements (which dominate the behavior of the lowfield susceptibility) are largely reversible.

The main graph in Fig. 10 depicts $\chi_{\text {ext }}^{\prime}(T)$ at 159 and 3000 $\mathrm{Hz}$, both with $H_{\mathrm{ac}}=1$ Oe. $\chi_{\mathrm{ext}}^{\prime}(T)$ exhibits virtually no dependence on frequency in the entire temperature range $5 \mathrm{~K}$ $\leq T \leq 320 \mathrm{~K}$, which is consistent with a bulk ferromagnetic behavior with no relaxational effects due to domain walls. The magnetocrystalline anisotropy of Gd is relatively low, so domain magnetization rotation probably also significantly contributes to $\chi_{\text {ext }}^{\prime}(T)$. The inset of Fig. 10 shows the variation in the imaginary (lossy) part of the susceptibility with temperature $\left[\chi_{\text {ext }}^{\prime \prime}(T)\right]$ for the same two frequencies. $\chi_{\text {ext }}^{\prime \prime}(T)$ is independent of frequency in the vicinity of $T_{C}$, as expected at a transition to long-range order; however, near $T_{\mathrm{SR}}$, the magnitude of $\chi_{\text {ext }}^{\prime \prime}(T)$ becomes frequency dependent, with the higher frequency having greater $\chi_{\mathrm{ext}}^{\prime \prime}(T)$. The rounded peaks in $\chi_{\text {ext }}^{\prime \prime}(T)$ occur at the same temperature $(\sim 180 \mathrm{~K}$ within the uncertainties) for both frequencies. This temperature is coincident with the temperature at which $\chi_{\mathrm{ext}}^{\prime}(T)$ begins to decrease with decreasing $T$. Interestingly, there is a sharp peak in $\chi_{\text {ext }}^{\prime \prime}(T)$ at $\sim 20 \mathrm{~K}$ for both frequencies. There is no appar- 


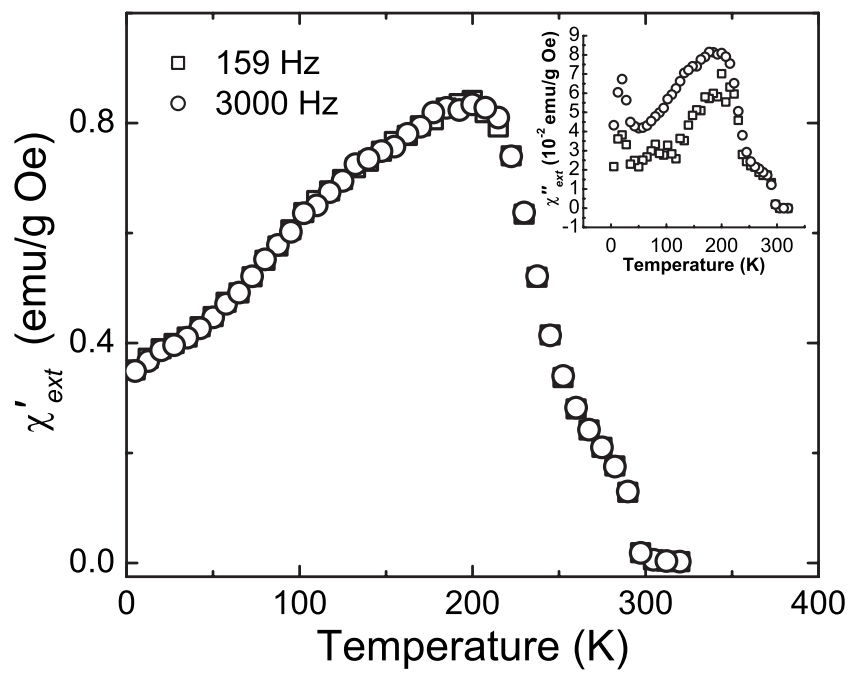

FIG. 10. Real part of the external susceptibility as a function of temperature $\chi_{\text {ext }}^{\prime}(T)$ for two different frequencies, 159 and $3000 \mathrm{~Hz}$. The ac driving field amplitude $H_{\mathrm{ac}}=1$ Oe. The inset shows the imaginary part $\chi_{\mathrm{ext}}^{\prime \prime}(T)$ for the same frequencies.

ent shift with frequency in the temperature at which the peak occurs. This sharp peak in $\chi_{\mathrm{ext}}^{\prime \prime}(T)$ at low temperature has not, to the best of our knowledge, been seen before in polycrystalline or monocrystalline Gd.

The behavior of $\chi_{\text {ext }}^{\prime}(T)$ at $3000 \mathrm{~Hz}$ in different dc bias fields is presented in Fig. 11, with the data taken after zerofield cooling. A relatively small bias field of 150 Oe dramatically diminishes $\chi_{\text {ext }}^{\prime}(T)$, which is attributable to the small coercivity of ms-Gd. Also, the zero-bias-field peak in $\chi_{\text {ext }}^{\prime}(T)$ is shifted to higher temperatures, which is the opposite response to that of the Hopkinson peak typically observed in ferromagnets. ${ }^{22}$ The shift to higher temperatures is due to the higher bias field pushing the collapse of the temperaturedependent moment toward higher temperatures as the

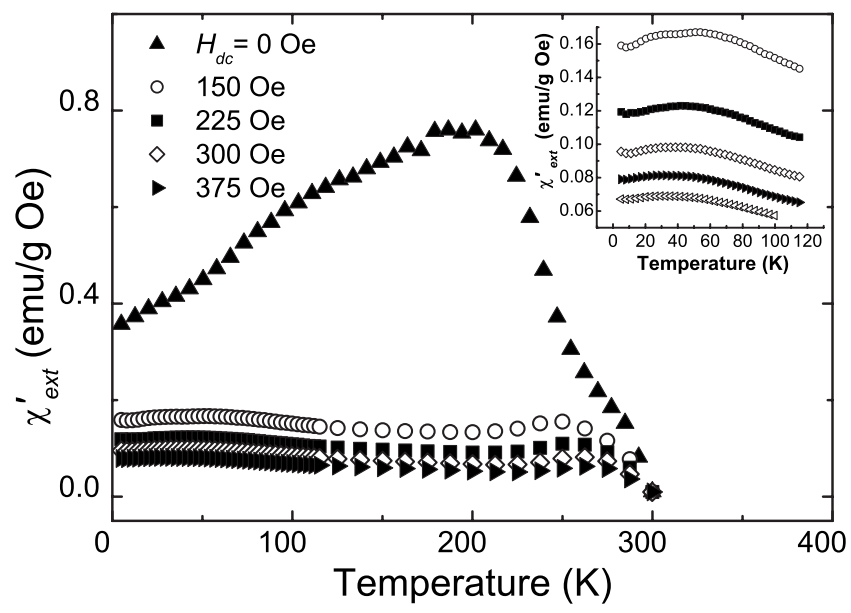

FIG. 11. Real part of the external ac susceptibility as a function of temperature $\chi_{\mathrm{ext}}^{\prime}(T)$ with various dc bias fields $\left(150\right.$ Oe $\leq H_{\mathrm{dc}}$ $\leq 450$ Oe) imposed. The frequency is $3000 \mathrm{~Hz}$. The $450 \mathrm{Oe}$ data are omitted from the main graph for clarity. The inset shows the low-temperature peak that appears for high-enough bias fields (including $450 \mathrm{Oe})$.

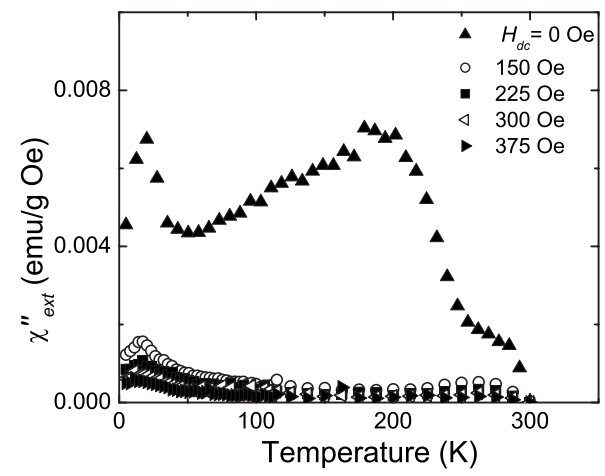

FIG. 12. Imaginary part of the external ac susceptibility as a function of temperature $\chi_{\text {ext }}^{\prime \prime}(T)$ with various dc bias fields (150 Oe $\leq H_{\mathrm{dc}} \leq 375$ Oe) imposed. The frequency is $3000 \mathrm{~Hz}$.

FM-PM transition is approached. This effect dominates the influence of the temperature-dependent anisotropy, which is near zero in the vicinity of the zero-bias-field peak and relatively weakly depends on temperature between the peak in $\chi_{\mathrm{ext}}^{\prime}(T)$ and $T_{C}$. With $H_{\mathrm{dc}} \approx 0, \chi_{\mathrm{ext}}^{\prime}(T)$ monotonically decreases with decreasing temperature beyond the peak, with a change in slope (the slope becomes shallower) at $\sim 50 \mathrm{~K}$. However, for the bias-field values shown in Fig. 11 $\left(150 \mathrm{Oe} \leq H_{\mathrm{dc}} \leq 450 \mathrm{Oe}\right)$, a second peak develops in the vicinity of the slope change in the zero-bias-field data. The inset of Fig. 11 shows the low-temperature peaks in more detail. The peaks are broad because of the overall suppression of $\chi_{\mathrm{ext}}^{\prime}(T)$ by the applied de field, which drives the system toward saturation. The temperature $T_{p}$ at which the peak occurs decreases as $H_{\mathrm{dc}}$ increases. This behavior was observed in SG, RSG, and paramagnets. ${ }^{19,20}$ The lowtemperature peaks are sharper in $\chi_{\text {ext }}^{\prime \prime}(T)$, as shown in Fig. 12. The suppressive effect of $H_{\mathrm{dc}}$ on $\chi_{\mathrm{ext}}^{\prime \prime}(T)$ is even greater than on $\chi_{\mathrm{ext}}^{\prime}(T)$; however, the behavior around the lowtemperature peak is quite distinct, despite the presence of $H_{\mathrm{dc}}$. The effect of $H_{\mathrm{dc}}$ on $\chi_{\mathrm{ac}}(T)$ at low temperatures is reminiscent of the data for $\chi_{\mathrm{ac}}(T)$ in the vicinity of the PM-FM transition presented earlier in Fig. 6, in which a dc bias field suppressed the technical contributions to the magnetization and uncovered the critical susceptibility maxima.

The ZFC magnetization as a function of field at $5 \mathrm{~K}$ is shown in Fig. 13. As seen more clearly in the inset of Fig. $13, M(H)$ has not reached saturation at $5 \mathrm{~K}$ and in a field of 70 kOe. Pure crystalline Gd has a relatively small magnetocrystalline anisotropy and, hence, a relatively small coercivity ( $\sim 15$ Oe at $77 \mathrm{~K}$ for polycrystalline $\mathrm{Gd}) \cdot{ }^{27}$ In pure $\mathrm{Gd}$ single crystals at $4.5 \mathrm{~K}$, saturation occurred at $\sim 8 \mathrm{kOe}$ with the field along the easy axis and $\sim 12 \mathrm{kOe}$ for the hard axis. $^{28}$

The failure to achieve saturation at $5 \mathrm{~K}$ and in fields up 70 kOe in ms-Gd suggests an enhanced anisotropy, so we attempted to fit the high-field $M(H)$ data with models that incorporate anisotropy. The random-anisotropy model of Chudnovsky, Saslow, and Serota $(\mathrm{CSS})^{29}$ at high fields and including a high-field susceptibility $\left(\chi_{\mathrm{hf}}\right)$ term predicts that the magnetization approaches saturation as 


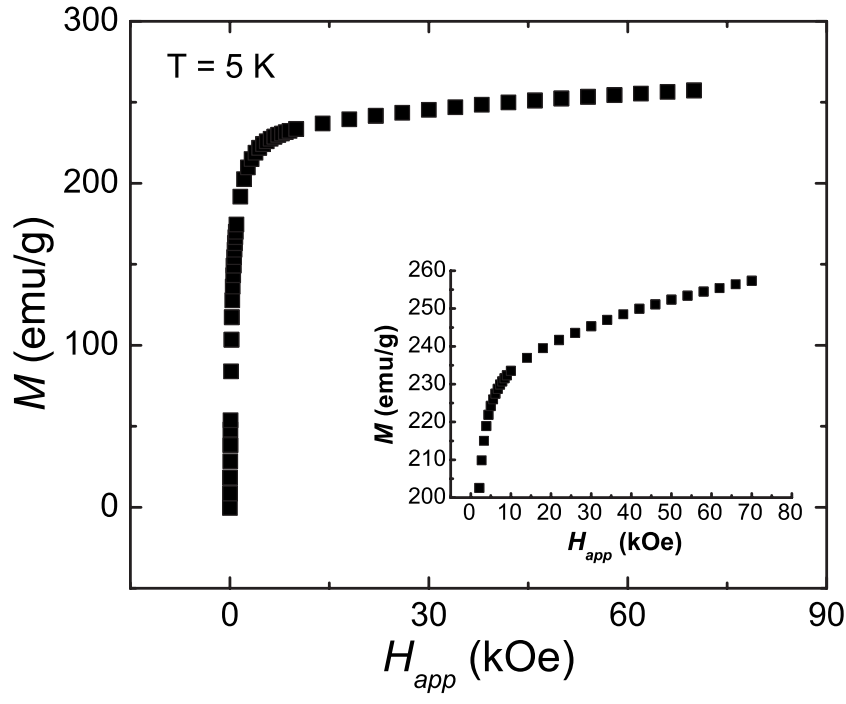

FIG. 13. Zero-field-cooled magnetization $M$ versus external magnetic field $H_{\text {ext }}$ at $T=5 \mathrm{~K}$. The inset shows the increasing magnetization at the highest fields.

$$
M=M_{0}\left[1-\frac{1}{15}\left(\frac{H_{r}}{H_{\mathrm{app}}+H_{\mathrm{ex}}}\right)^{2}\right]+\chi_{\mathrm{hf}} H_{\mathrm{app}},
$$

where $M_{0}$ is the saturation magnetization, $H_{r}$ is an anisotropy field, $H_{\mathrm{ex}}$ is an exchange field, and $H_{\mathrm{app}}$ is the applied field. The second model used for the approach to saturation is

$$
M=M_{0}\left(1-b / H_{\mathrm{app}}^{2}\right)+\chi_{\mathrm{hf}} H_{\mathrm{app}}
$$

where $b$ is related to the anisotropy constant. ${ }^{30} \mathrm{~A}$ third model assumes two components, one of which is saturated (crystalline grains), while the other is unsaturated and described by the CSS random-anisotropy model. The volume fraction of each component was obtained from the EXAFS measurements.

The fitting in each case was performed by using a "rangeof-fit" technique in which the range of field values included in the fit was changed and the variation in the fit parameters observed. The single-component model described by Eq. (8) gave the best fit in terms of low chi-squared value and constancy of the parameters as the range of the fit was varied. For $14 \mathrm{kOe} \leq H_{\mathrm{app}} \leq 70 \mathrm{kOe}, M_{0}=257 \mathrm{emu} / \mathrm{g}$ (7.24 $\mu_{B}$ /atom), $H_{r}=88.0 \mathrm{kOe}, H_{\mathrm{ex}}=64.8 \mathrm{kOe}$, and $\chi_{\mathrm{hf}}$ $=1.12 \times 10^{-4} \mathrm{emu} / \mathrm{g} \cdot$ Oe. The data and best-fit line are shown in Fig. 14. $M_{0}$ obtained from the fit is lower than values for Gd single crystals [e.g., 7.55 $\mu_{B}$ /atom (Ref. 31)] but is still significantly greater than the spin-only Gd moment. The anisotropy field is greater than the maximum applied field of $70 \mathrm{kOe}$, which contravenes the requirement $H_{\text {app }} \gg H_{r}$; however, as observed by Gehring et al.,${ }^{32}$ the prefactor 1/15 may have to be modified when Eq. (8) is applied to real materials. If the prefactor is changed to $1, H_{r}$ falls to $23 \mathrm{kOe}$. The requirement $H_{\mathrm{app}}>H_{\mathrm{ex}}$ for the validity of Eq. (8) was strictly met only by our highest applied field (70 kOe); however, the fit is good enough to suggest that the applicability of Eq. (8) may extend to applied fields smaller than $H_{\mathrm{ex}}$.

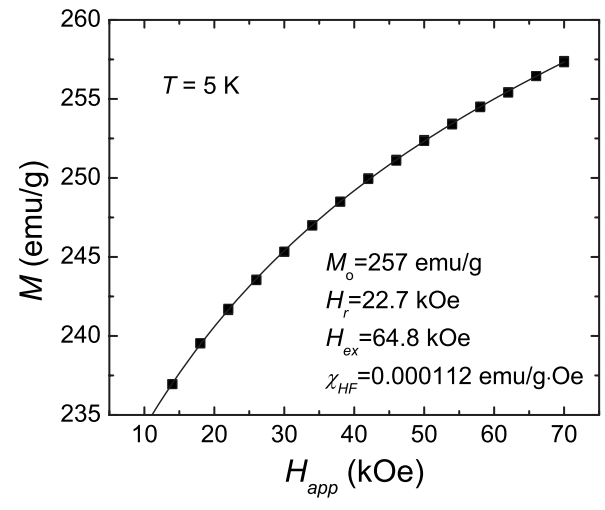

FIG. 14. High-field de magnetization data $(T=5 \mathrm{~K})$ and fit obtained by using the high-field expression for the random-anisotropy model of Chudnovsky et al. (Ref. 29) (see text). The best-fit parameters are shown in the figure.

\section{DISCUSSION}

\section{Paramagnetic-to-ferromagnetic transition}

The susceptibility of ms-Gd follows a Curie-Weiss law for $T>T_{C}$, indicating that no significant atomic clustering was induced in the grains or intergrain regions due to rapid quenching during melt spinning. The $M(T)$ data exhibit a slight upward curvature, so our experimental $p_{\text {eff }}=8.14$ is an overestimate and its true value is somewhat closer to $p_{\text {eff }}$ $=7.98$ found in bulk crystalline Gd. ${ }^{33}$ The curvature in $1 / \chi$ versus $T$ for temperatures up to $20 \%$ greater than $T_{C}$ (which also was observed in bulk crystalline Gd) is likely an indication of the persistence of local ferromagnetic correlations at $T>T_{C}$.

Our value of $T_{C}=289.70 \pm 0.01 \mathrm{~K}$ is approximately $1.5 \mathrm{~K}$ lower than that for a polycrystalline sample and approximately $3 \mathrm{~K}$ lower than that measured for single crystals. ${ }^{14,34}$ We therefore posit that the depression in $T_{C}$ is due to the nanocrystallinity of our Gd sample. For Gd grain sizes between 10 and $100 \mathrm{~nm}$, Michels et al. ${ }^{24}$ found a power-law relationship between $T_{C}$ (relative to coarse-grained $\mathrm{Gd}$ ) and grain size $D:-\Delta T_{C} \propto D^{-1}$. A change in $T_{C}$ of $\sim 10 \mathrm{~K}$ would be expected for a grain size of $24 \mathrm{~nm}$, which is somewhat greater than our result. Michels et al. ${ }^{24}$ attributed the reduction in $T_{C}$ with grain size to the pressure that is internally applied to the grain by the surrounding grain boundaries. $T_{C}$ for bulk crystalline $\mathrm{Gd}$ decreases with externally applied pressure. ${ }^{35}$ This can be explained in terms of a simplified Ruderman-Kittel-Kasuya-Yosida (RKKY) picture in which the change in the density of states at the Fermi level brought about by the applied pressure decreases the dominant exchange integral. EXAFS measurements on our ms-Gd sample indicated that the nearest-neighbor coordination radius is smaller than that for bulk crystalline $\mathrm{Gd}$ by $2.5 \%$, which is consistent with the expected effect of pressure. ${ }^{11} \mathrm{We}$ note, however, that recent $a b$ initio calculations ${ }^{36}$ showed that $T_{C}$ is rather sensitive to the $c / a$ ratio and that furtherneighbor interactions are also important in predicting the correct value of $T_{C}$. Our EXAFS data did not provide information on the $c / a$ ratio or changes in coordination distances beyond the first shell. 
The precise nature of the PM-FM transition is characterized by critical exponents and amplitudes, and determining the effect of structural inhomogeneity on the nature of the PM-FM transition was a primary goal of this study. Kaul and co-workers ${ }^{37,38}$ showed that single-crystal Gd undergoes a series of crossovers as the critical point is approached from above or below. When $T_{C}$ is approached from below, there is a crossover from the $3 \mathrm{D}$ isotropic short-range Heisenberg (ISH) universality class to the 3D uniaxial dipolar (UD) universality class. When $T_{C}$ is approached from above, there is first a slow crossover from an assumedly Gaussian regime to a 3D isotropic dipolar (ID) behavior. As $T_{C}$ is approached more closely, there is a sharp crossover from an ID behavior to a UD behavior. Renormalization-group calculations predict that if the long-range dipolar interactions are weak enough, then there is an additional crossover to a 3D ISH behavior between the Gaussian and ID regimes. The exponents for the 3D ISH and ID systems are quite similar (e.g., $\gamma_{\mathrm{ISH}}=1.386$ and $\left.\gamma_{\mathrm{ID}}=1.372\right),{ }^{14,39}$ so distinguishing between the two regimes requires consideration of the universal critical-amplitude ratios. ${ }^{37}$

For the range of $\varepsilon$ values of our measurements on ms-Gd, the effective critical exponents $\gamma_{\text {eff }}$ and $\beta_{\text {eff }}$ are nearly constant within the uncertainty of the data. The value $\beta_{\text {eff }}$ $=0.389 \pm 0.017\left(2.4 \times 10^{-3} \leq|\varepsilon| \leq 1.3 \times 10^{-2}\right)$ we have obtained compares very well to $\beta_{\text {eff }}=0.40 \pm 0.02\left(2.1 \times 10^{-3}\right.$ $\leq|\varepsilon| \leq 1.1 \times 10^{-2}$ ) obtained by Srinath and $\mathrm{Kaul}^{37}$ outside the asymptotic regime in which the critical behavior is driven by UD interactions. Further, Srinath and Kaul (SK) obtained $\gamma_{\text {eff }}=1.39 \pm 0.03$ in the reduced-temperature range 2.0 $\times 10^{-3} \leq \varepsilon \leq 6.8 \times 10^{-3}$. This seemingly disagrees with the value $\gamma_{\mathrm{eff}}=1.300 \pm 0.014$ obtained in this work; however, the reduced-temperature range for our determination was $1.0 \times 10^{-3} \leq \varepsilon \leq 1.5 \times 10^{-2}$, which encompasses the region covered by SK's single power-law fit and, at the low end, contains the region within which $\gamma_{\text {eff }}$ rapidly falls as the crossover to UD behavior is approached. It is thus reasonable that our $\gamma_{\text {eff }}$ value is somewhat smaller than SK's value and suggests that at our smallest value of $\varepsilon$, the ms-Gd system is close to the ID $\rightarrow \mathrm{UD}$ crossover. Moreover, in the $\ln M_{S}\left(\chi_{i}^{-1}\right)$ versus $\ln \varepsilon$ graph from which $\beta_{\text {eff }}\left(\gamma_{\text {eff }}\right)$ was determined, there is a slight but systematic curvature. This suggests that the addition of correction-to-scaling (CTS) terms to the single power-law relations for the critical exponents is necessary to obtain asymptotic values of the exponents. This is supported by the fact that Aliev et al. ${ }^{39}$ obtained $\gamma_{\text {eff }}$ $=1.33 \pm 0.02$ for monocrystalline $\mathrm{Gd}$, which agrees, within uncertainty, with our value for ms-Gd. When they employed CTS, they obtained an asymptotic $\gamma$ value of $1.39 \pm 0.02$, which is in accord with the theoretical exponent for an isotropic dipolar system. (Our data set is too limited to attempt fits with the greater number of free parameters necessary with a CTS expression.)

The critical amplitudes as defined in Eqs. (2) and (3) are effective amplitudes because of the omission of correctionto-scaling terms. After evaluating these amplitudes from the same log-log plots used to determine the critical exponent values, we find $B_{\text {eff }}=1.07 \pm 0.09, \Gamma_{\text {eff }}=0.24 \pm 0.03$, and $D$ 2.7. To calculate these values, we have used $M_{0}$ $=257 \mathrm{emu} / \mathrm{g}$, which was determined from fitting $M(H)$ at 5
$\mathrm{K}$ with Eq. (8). With the exception of $\delta$ and $D$, all of the critical amplitudes and exponents that we have determined for ms-Gd agree, within uncertainty limits, with the effective values obtained for single-crystalline Gd by Aliev et al. ${ }^{39}$ As noted by $\mathrm{SK}$, the determination of $\delta$ and $D$ are sensitive to the range of fields used. We used internal fields up to $60 \mathrm{kOe}$, while Aliev et al. used fields of up to $5 \mathrm{kOe}$. In addition, the uniaxial anisotropy field will add to the total field experienced by a spin. SK included the anisotropy field (which is dependent on the applied external magnetic field) in their determination of $\delta$ and the corresponding critical amplitude $\hat{D} .{ }^{37}$ Augmentation of the internal field with an anisotropy field in our case would tend to increase our value of $D$, bringing it closer to the 6.72 value obtained by Aliev et al. This would also bring the value of the universal criticalamplitude ratio $R_{\chi}=\Gamma D B^{\delta-1}$ for ms-Gd closer to the value of 1.33 for three-dimensional isotropic dipolar and Heisenberg systems. Given the larger applied fields that were used in our measurements, our results suggest that a larger anisotropy field than that in single-crystalline $\mathrm{Gd}$ is present in ms-Gd. This is reinforced by the $\chi_{\mathrm{ac}}(H, T)$ determination of the exponent $\gamma_{\mathrm{eff}}$, in which data at the lowest values of external bias field $\left(H_{\mathrm{dc}}<1000 \mathrm{Oe}\right)$ produced significant deviations from the linear fit whose slope equals $\gamma_{\text {eff }}$. Again, the addition of an anisotropy field to the applied field would tend to improve the linearity over the entire range of applied fields. Hence, for the range of $\varepsilon$ used in this work, the critical behavior of ms-Gd at the PM-FM transition is consistent with that of the isotropic dipolar universality class, which is the same behavior exhibited by monocrystalline Gd. We conclude that the structural inhomogeneity appears to have little effect on the universality class of the PM-FM transition, although $T_{C}$ is depressed and there is evidence of enhanced anisotropy.

\section{Ferromagnetic regime}

The absence of significant ac field (up to $1 \mathrm{Oe}$ ) or frequency (up to $3000 \mathrm{~Hz}$ ) dependence in $\chi_{\text {ext }}^{\prime}(T)$ in zero dc bias field for $T<T_{C}$ indicates that the dynamics is governed by nearly relaxation-free reversible domain-wall displacements. $\chi_{\text {ext }}^{\prime}(T)$ displays a clear frequency dependence in many ferromagnetic systems that is usually attributed to relaxation due to energy barriers created by domain-wall pinning. In disordered ferromagnets at low temperatures, such relaxation may arise due to a cluster-glass behavior. The absence of frequency dependence in $\chi_{\text {ext }}^{\prime}(T)$-even at the lowest temperatures - suggests that domain walls are trapped within pinning-potential wells, many of which are likely located in the grain-boundary regions. Domain magnetization rotation (DMR) thus probably significantly contributes to $\chi_{\text {ext }}^{\prime}(T)$.

$\chi_{\text {ext }}^{\prime \prime}(T)$ does show some frequency dependence and $\chi_{\text {ext }}^{\prime \prime}(T) / \chi_{\text {ext }}(T) \approx 10^{-2}$, which is significantly smaller than in many ferromagnetic systems that show frequency dependence of $\chi_{\text {ext }}^{\prime}(T) \cdot{ }^{10,40,41}$ Estimation of eddy-current effects ${ }^{41}$ gives $\chi_{e \text {,eddy }}^{\prime \prime} \approx 2 \chi_{\text {ext }}^{\prime \prime}$ for $f=3000 \mathrm{~Hz}$, but $\chi_{e, \text { eddy }}^{\prime \prime} \approx 0.2 \chi_{\text {ext }}^{\prime \prime}$ for $f=159 \mathrm{~Hz}$; however, the estimate uses the resistivity of a single crystal $^{33}$ and assumes uniform magnetization. The most robust conclusion we can make is that eddy-current effects are significant at $3000 \mathrm{~Hz}$ but not at $159 \mathrm{~Hz}$. Account- 
ing for eddy-current contributions would decrease the frequency dependence of the magnitude of $\chi_{\mathrm{ext}}^{\prime \prime}(T)$, making it more similar to that of $\chi_{\mathrm{ext}}^{\prime}(T)$.

The suppression of the contribution of domain walls and weak-field DMR at all $T<T_{C}$ by applying an external bias field reveals a low-temperature peak in $\chi_{\text {ext }}^{\prime}(T)$. For $T$ $<120 \mathrm{~K}$, the diminution of the temperature at which the peak in $\chi_{\text {ext }}^{\prime}(T)$ occurs with increasing bias field, the bifurcation in ZFC and FC magnetization, and the occurrence of a relatively sharp zero-bias-field peak in $\chi_{\mathrm{ext}}^{\prime \prime}(T)$ taken together suggest a crossover of the system from a pure FM state at high temperatures to a cluster-glass-like (CGL) state at low temperatures. Like amorphous ${ }^{42}$ and substitutionally disordered crystalline ${ }^{43}$ ferromagnets, this CGL state is driven by anisotropy, which increases in strength as the temperature decreases. The peak appears in $\chi_{\text {ext }}^{\prime}(T)$ because a given static bias field has less effect on $\chi_{\text {ext }}^{\prime}(T)$ at lower temperatures at which the anisotropy is higher. The greater suppression of $\chi_{\mathrm{ext}}^{\prime}(T)$ at higher temperatures and the small values of $\chi_{\mathrm{ext}}^{\prime}(T)$ at the lowest temperatures lead to a maximum at intermediate temperatures. As the static bias field is increased, higher anisotropy values are required to counteract the suppressive effect of the field on $\chi_{\text {ext }}^{\prime}(T)$, so the peak occurs at lower temperatures.

The distinct peak in $\chi_{\text {ext }}^{\prime \prime}(T)$ certainly calls to mind a sharper "re-entrant" transition to a low-temperature "frozen" state; however, the absence of frequency dependence in the zero-bias-field $\chi_{\text {ext }}^{\prime}(T)$ data militates against a re-entrant transition to a typical cluster-glass state with a wide distribution of cluster sizes and relaxation times. Moreover, re-entrant transitions are usually accompanied by a sharp downturn in $\chi^{\prime}(T)$, which is not observed in our data. In fact, the downslope in $\chi_{\mathrm{ext}}^{\prime}(T)$ becomes somewhat shallower at low temperatures. There also is no sharp increase in the coercivity of ms-Gd down to $40 \mathrm{~K}$ (which was the lowest temperature at which the data were taken). ${ }^{11}$ Thus, the low-temperature state in ms-Gd is unlike the cluster-glass or mixed magnetic states observed in amorphous ferromagnets.

We attribute the previously unobserved low-temperature peak in $\chi_{\mathrm{ext}}^{\prime \prime}(T)$ to the decoupling of the grains due to increased random anisotropy in the intergrain regions. As pointed out before, this magnetization change is not due to relaxation processes as there is no frequency dependence. It is also not likely to be due to eddy currents because there is no sharp change in the resistivity of $\mathrm{Gd}$ at low temperatures ${ }^{33}$ and, furthermore, there is no accompanying anomaly in $\chi_{\text {ext }}^{\prime}(T)$. We propose that the low-temperature anomaly in $\chi_{\mathrm{ext}}^{\prime \prime}(T)$ is due to the frustration of the ferromagnetic coupling of the grains due to increased random anisotropy at low temperatures within the disordered intergrain regions and the resulting lowering of exchange penetration of the boundaries. Dipole-dipole interactions also tend to reduce ferromagnetic correlations between domains on either side of a grain boundary. This decoupling of the relatively large grains (grain diameter $>$ exchange length) has little effect on the zero-field behavior of $\chi_{\text {ext }}^{\prime}(T)$, which is determined by domain magnetization rotation and domain-wall displacement within the multidomain grains. However, because of the small magnitude of $\chi_{\mathrm{ext}}^{\prime \prime}(T)$, the small magnetization change per cycle of the ac field induced by the inhibited magnetic coupling clearly manifests as a peak in $\chi_{\mathrm{ext}}^{\prime \prime}(T)$.

Additional evidence for the inhibited magnetic coupling of the grains is found in the approach to saturation at very low temperatures $(5 \mathrm{~K})$. Decreased correlation between grains should inhibit full saturation, as is observed. The large external applied fields produce single-domain grains; however, the magnetizations of the grains do not completely align in fields of up to $70 \mathrm{kOe}$ because of the disordered intergrain regions and associated random anisotropy. It is therefore fitting that the random-anisotropy model best describes the approach to saturation. By using the best-fit value $H_{r}=23 \mathrm{kOe}$, we estimate ${ }^{44}$ the random anisotropy strength to be $K_{r}=M_{0} H_{r} / 2 \sim 2 \times 10^{7} \mathrm{erg} / \mathrm{cm}^{3}$, which is approximately an order of magnitude larger than the magnetocrystalline anisotropy constant of monocrystalline Gd. This anisotropy enhancement may be similar in origin to that seen in metal nanoparticles, in which the localization of $d$ electrons at the surfaces gives rise to increased anisotropy. ${ }^{45,46}$

For applied fields in the range $H_{r}<H_{\text {app }}<H_{\text {ex }}$, the CSS random anisotropy model predicts a field-dependent ferromagnetic correlation length (FCL) $R_{F}^{\perp}=R_{a}\left(H_{\text {ex }} / H_{\text {app }}\right)^{1 / 2}$, where $R_{a}$ is the distance over which the local anisotropy axes are correlated. ${ }^{29}$ If the weak anisotropy condition $\left(H_{r}\right.$ $\left.\ll H_{\text {ex }}\right)$ is satisfied, then $R_{F}^{\perp} \gg R_{a}$ and the $\left(H_{s} / H_{\text {app }}\right)^{1 / 2}$ approach-to-saturation law is obtained. Our best-fit values for $H_{r}$ and $H_{\mathrm{ex}}$ (23 and $65 \mathrm{kOe}$, respectively) indicate that the weak random anisotropy condition is not satisfied. In fact, for the field range over which our data were fit, the FCL falls in the range $0.96 R_{a}<R_{F}^{\perp}<2.1 R_{a}$. In other words, $R_{F}^{\perp} \approx R_{a}$, which is a condition characteristic of the high-field regime, where the $\left[H_{r} /\left(H_{\mathrm{app}}+H_{\mathrm{ex}}\right)\right]^{2}$ approach-to-saturation law [Eq. (8)] is valid. The excellent description of our data by this law is therefore a result of the relatively high anisotropy of msGd.

In the CSS model, the anisotropy correlation length $R_{a}$ is taken to be a few atomic spacings $(a)$ for amorphous materials and $R_{a} \gg a$ for polycrystalline materials. In ms-Gd, the FCL is likely limited by the grain size. Thus, $R_{a}$ should be smaller than the average grain size since $R_{F}^{\perp}>R_{a}$ for most of the fields used in our approach-to-saturation data and the best fit. A value of $R_{a}$ smaller than the grain size could result from strain-induced fluctuations in the anisotropy on scales smaller than the grain size. ${ }^{47}$

We conclude that melt-spun Gd consists of multidomain grains magnetically coupled by exchange at temperatures from $T_{C}$ down to $\sim 100 \mathrm{~K}$. Below the spin-reorientation temperature, the increase in anisotropy strength (predominantly random anisotropy in the intergrain regions) leads to an irreversibility as manifested in the ZFC and FC magnetizations. The increasing strength of the random anisotropy in the intergrain regions decreases the magnetic coupling of the grains, which inhibits saturation of the sample at low temperatures and high fields.

\section{CONCLUSIONS}

Melt-spun Gd is a structurally inhomogeneous system consisting of crystalline grains with an average size of $24 \pm 3 \mathrm{~nm}$ that are separated by noncrystalline intergrain re- 
gions. The grain boundaries compress the grains, leading to a depression of $T_{C}(289.70 \pm 0.01 \mathrm{~K})$ relative to bulk Gd (293 $\mathrm{K})$; however, the effective critical exponents ( $\beta_{\text {eff }}$ $=0.389 \pm 0.017, \gamma_{\text {eff }}=1.300 \pm 0.014$, and $\delta=4.32 \pm 0.02$ ) and amplitudes indicate that for the reduced-temperature range of this work, the nature of the PM-FM transition is consistent with the isotropic dipolar universality class, which is the same behavior displayed by structurally homogeneous $\mathrm{Gd}$. The critical exponent $\delta$ and its associated critical amplitude $D$ are, however, different from the values obtained in monocrystalline Gd. These two parameters are more sensitive to anisotropy and the discrepancies suggest additional anisotropy in ms-Gd. The enhanced intergrain anisotropy is supported by the behavior observed below $T_{C}$. Increasing random anisotropy in the grain-boundary and intergrain regions with decreasing temperature diminish the magnetic coupling between the grains and produce a previously unseen low-temperature peak in $\chi_{\text {ext }}^{\prime \prime}(T)$. The random anisotropy model provides a good description of the approach to satu- ration. The applicability of this model may be initially surprising because of the relatively large size of the grains; however, it should be noted that in the strong-field regime of the model, the ferromagnetic correlation length becomes comparable to the anisotropy correlation length. This condition is likely to be satisfied in ms-Gd.

\section{ACKNOWLEDGMENTS}

This work was supported by National Science Foundation Grant Nos. DMR-0504706 and DMR-05-04177. EXAFS measurements were made at the Advanced Photon Source at Argonne National Laboratory, which is supported by the U.S. Department of Energy, Office of Science, under Contract No. DE-AC02-06CH11357. The authors thank Daniel Haskel for assistance in acquiring the EXAFS data. One of the authors (P.M.S.) would like to thank Nick Jensen for assistance with the preparation of graphs.
${ }^{1}$ K. Suzuki and J. M. Cadogan, Phys. Rev. B 58, 2730 (1998).

${ }^{2}$ T. Kaneyoshi, Introduction to Amorphous Magnets (World Scientific, Singapore, 1992).

${ }^{3}$ Y. Kakehashi, T. Uchida, and M. Yu, Phys. Rev. B 56, 8807 (1997).

${ }^{4}$ Properties and Applications of Nanocrystalline Alloys from Amorphous Precursors, edited by B. Idzikowski, P. Svec, and M. Miglierini (Kluwer, Dordrecht, 2005).

${ }^{5}$ A. Hernando, E. Navarro, M. Multigner, A. R. Yavari, D. Fiorani, M. Rosenberg, G. Filoti, and R. Caciuffo, Phys. Rev. B 58, 5181 (1998).

${ }^{6}$ L. Del Bianco, A. Hernando, E. Bonetti, and E. Navarro, Phys. Rev. B 56, 8894 (1997).

${ }^{7}$ E. Bonetti, L. Del Bianco, D. Fiorani, D. Rinaldi, R. Caciuffo, and A. Hernando, Phys. Rev. Lett. 83, 2829 (1999).

${ }^{8}$ G. Alvarez and E. Dagotto, J. Magn. Magn. Mater. 272-276, 15 (2004).

${ }^{9}$ Nanomaterials: Synthesis, Properties, and Applications, edited by A. S. Edelstein and R. C. Cammarata (IOP, Bristol, 1996).

${ }^{10}$ S. N. Kaul and S. Srinath, Phys. Rev. B 62, 1114 (2000).

${ }^{11}$ D. Schmitter, Ph.D. thesis, University of Nebraska-Lincoln, 2007.

${ }^{12}$ A. Arrott and J. E. Noakes, Phys. Rev. Lett. 19, 786 (1967).

${ }^{13}$ A. Pelissetto and E. Vicari, Phys. Rep. 368, 549 (2002).

${ }^{14}$ S. N. Kaul, J. Magn. Magn. Mater. 53, 5 (1985).

${ }^{15} \mathrm{H}$. E. Stanley, Introduction to Phase Transitions and Critical Phenomena (Oxford University Press, New York, 1971).

${ }^{16}$ W. Li, H. P. Kunkel, X. Z. Zhou, G. Williams, Y. Mukovskii, and D. Shulyatev, Phys. Rev. B 70, 214413 (2004).

${ }^{17}$ J. H. Zhao, H. P. Kunkel, X. Z. Zhou, G. Williams, and M. A. Subramanian, Phys. Rev. Lett. 83, 219 (1999).

${ }^{18}$ X. Zhou, W. Li, H. P. Kunkel, and G. Williams, Phys. Rev. B 73, 012412 (2006).

${ }^{19}$ K. H. Fischer and J. Hertz, Spin Glasses (Cambridge University Press, Cambridge, 1991).

${ }^{20}$ J.-L. Tholence, in Magnetic Susceptibility of Superconductors and Other Spin Systems, edited by R. A. Hein, T. L. Francavilla, and D. H. Liebenberg (Plenum, New York, 1991).

${ }^{21}$ P. A. Joy, P. S. Kumar, and S. K. Date, J. Phys.: Condens. Matter 10, 11049 (1998).

${ }^{22}$ A. Senchuk, H. P. Kunkel, R. M. Roshko, C. Viddal, L. Wei, G. Williams, and X. Z. Zhou, Eur. Phys. J. B 37, 285 (2003).

${ }^{23}$ X. L. Wang, J. Horvat, H. K. Liu, and S. X. Dou, Solid State Commun. 108, 661 (1998).

${ }^{24}$ D. Michels, C. E. Krill III, and R. Birringer, J. Magn. Magn. Mater. 250, 203 (2002).

${ }^{25}$ F. Milstein and L. B. Robinson, Phys. Rev. 177, 904 (1969).

${ }^{26}$ J. M. D. Coey, V. Skumryev, and K. Gallagher, Nature (London) 401, 35 (1999).

${ }^{27}$ L. F. Bates and A. J. Pacey, Proc. Phys. Soc. Jpn. 78, 878 (1961).

${ }^{28}$ S. Y. Dan'kov, A. M. Tishin, V. K. Pecharsky, and K. A. Gschneidner, Phys. Rev. B 57, 3478 (1998).

${ }^{29}$ E. M. Chudnovsky, W. M. Saslow, and R. A. Serota, Phys. Rev. B 33, 251 (1986).

${ }^{30}$ A. H. Morrish, The Physical Properties of Magnetism (Wiley, New York, 1965).

${ }^{31}$ M. D. Kuz'min, A. S. Chernyshov, V. K. Pecharsky, K. A. Gschneidner, Jr., and A. M. Tishin, Phys. Rev. B 73, 132403 (2006).

${ }^{32}$ P. M. Gehring, M. B. Salamon, A. del Moral, and J. I. Arnaudas, Phys. Rev. B 41, 9134 (1990).

${ }^{33}$ H. E. Nigh, S. Legvold, and F. H. Spedding, Phys. Rev. 132, 1092 (1963).

${ }^{34}$ P. Hargraves, R. A. Dunlap, D. J. W. Geldart, and S. P. Ritcey, Phys. Rev. B 38, 2862 (1988).

${ }^{35}$ M. Tokita, K. Zenmyo, H. Kubo, K. Takeda, M. Mito, and T. Iwamoto, J. Magn. Magn. Mater. 272, 593 (2004).

${ }^{36}$ I. Turek, J. Kudrnovsky, G. Bihlmayer, and S. Blugel, J. Phys.: Condens. Matter 15, 2771 (2003).

${ }^{37}$ S. Srinath and S. N. Kaul, Phys. Rev. B 60, 12166 (1999).

${ }^{38}$ S. Srinath, S. N. Kaul, and H. Kronmüller, Phys. Rev. B 59, 
1145 (1999).

${ }^{39}$ K. H. K. Aliev, I. K. Kamilov, and A. M. Omarov, Zh. Eksp. Teor. Fiz. 94, 153 (1988).

${ }^{40}$ D. X. Chen, V. Skumryev, and J. M. D. Coey, Phys. Rev. B 53, 15014 (1996).

${ }^{41}$ D. X. Chen, V. Skumryev, and H. Kronmuller, Phys. Rev. B 46, 3496 (1992).

${ }^{42}$ S. N. Kaul and S. Srinath, J. Phys.: Condens. Matter 10, 11067 (1998).

${ }^{43}$ P. A. Joy and S. K. Date, J. Magn. Magn. Mater. 220, 106 (2000).
${ }^{44}$ D. J. Sellmyer and S. Nafis, J. Appl. Phys. 57, 3584 (1985).

${ }^{45}$ F. Luis, J. M. Torres, L. M. García, J. Bartolomé, J. Stankiewicz, F. Petroff, F. Fettar, J. L. Maurice, and A. Vaurès, Phys. Rev. B 65, 094409 (2002).

${ }^{46}$ M. Colarieti-Tosti, S. I. Simak, R. Ahuja, L. Nordström, O. Eriksson, D. Åberg, S. Edvardsson, and M. S. S. Brooks, Phys. Rev. Lett. 91, 157201 (2003).

${ }^{47}$ J. Weissmüller, A. Michels, D. Michels, A. Wiedenmann, C. E. Krill III, H. M. Sauer, and R. Birringer, Phys. Rev. B 69, 054402 (2004). 